\title{
Lysozyme and bovine serum albumin partitioning in polyethylene glycol-phenylalanine conjugate polymer/salt aqueous two-phase systems
}

\author{
S.C. Silvério ${ }^{a, b}$, L.A. Ferreira ${ }^{a, *}$, J.A. Martins ${ }^{c}$, J.C. Marcos $^{c}$, E.A. Macedo ${ }^{b}$, J.A. Teixeira ${ }^{a}$ \\ a IBB - Institute for Biotechnology and Bioengineering, Centre for Biological Engineering, Universidade do Minho, Campus de Gualtar, $4710-057$ Braga, Portugal

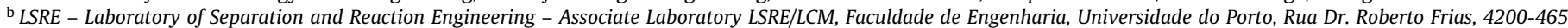 \\ Porto, Portugal \\ c Centro de Química, Universidade do Minho, Campus de Gualtar, 4710-057 Braga, Portugal
}

\section{A R T I C L E I N F O}

\section{Article history:}

Received 29 July 2011

Received in revised form 4 February 2012

Accepted 1 March 2012

Available online 8 March 2012

\section{Keywords:}

Aqueous two-phase systems

Derivatized polyethylene glycol

Protein partitioning

Protein surface hydrophobicity

\begin{abstract}
A B S T R A C T
Polyethylene glycol (PEG) with average molecular weight of 8000 was used as a model polymer and modified by introducing an amino acid (phenylalanine) in its hydroxyl terminals. The effect of different percentages of the conjugate polymer in relation to the total polymer on binodal curve of PEG-8000-potassium phosphate buffer (KPB), pH 7.2 aqueous two-phase systems and solute partitioning was explored. The partition of lysozyme (Lyz) and bovine serum albumin (BSA) and also of the conjugated polymer was evaluated in ATPS composed of $17 \%(\mathrm{w} / \mathrm{w})$ total polymer and $12.5 \%(\mathrm{w} / \mathrm{w}) \mathrm{KPB}, \mathrm{pH} 7.2$. Lyz partition profile follows the expected trend decreasing with the increase of modified polymer concentration. However BSA shows a partition profile that is approximately symmetrical to the one observed for the partition of the conjugate polymer, which depends on PEG-phenylalanine concentration in the system.

Determination of the relative hydrophobicity of the equilibrium phases and of the protein surface hydrophobicity (So) delivered information that shed some light in the mechanisms controlling the partitioning behavior. Therefore, it becomes clear that the hydrophobic character of the protein taken together with the molecular weight explain the different behavior of these two proteins with the increase of PEG-phenylalanine substitution in the systems.
\end{abstract}

(c) 2012 Elsevier B.V. All rights reserved.

\section{Introduction}

Purification of biomolecules, specially proteins, continues to be an important task both at the fundamental and applied levels. Unraveling the complexity of living systems often includes the characterization of the structure and function of isolated biomolecules. With the advent of -omics approaches the need for better and simple methods for isolating biomolecules had increased. On the other hand, the increasing number of approved biopharmaceuticals that need to be produced in large-scale stimulated the development of scalable and efficient processes for their purification. Although, in both cases the several types of chromatography continue to have a leading role, other methodologies are emerging as serious and advantageous alternatives.

One of them is liquid-liquid extraction using aqueous twophase systems (ATPS) that are formed when two polymers or a polymer and a salt are mixed in water above some critical concentrations. The advantages of ATPS include its operational simplicity,

\footnotetext{
* Corresponding author. Tel.: +351 253604420; fax: +351253678986

E-mail address: laferreira@deb.uminho.pt (L.A. Ferreira).
}

easy scale-up and down, possibility of continuous operation, and mild conditions of the phases.

In fact, they had been successfully used in different applications such as: large-scale separation and production of engineered proteins [1], multi-stage equilibrium aqueous two-phase extraction of human antibodies [2], initial primary downstream processing for partial purification of industrial enzymes [3] or separation of mixtures containing optical isomers or saccharides [4]. There are also some examples where recovery and purification using ATPS presents economical and technical advantages over traditional processes. A direct comparison between ATPS and chromatography showed that ATPS can be more efficient, providing high percentage of recovered product [3]. Additionally, ATPS can reduce both the number of steps and the costs associated with the purification process, when compared to chromatography [5,6].

Nevertheless, its wider application had been hindered by the limited understanding of phase equilibrium and solute partitioning. From the data gathered in several studies it is known that the protein partition between the two phases depends on its physicochemical properties such as size, isoelectric point, and surface hydrophobicity. Also, the characteristics of the ATPS such as the $\mathrm{pH}$, type and amount of salt, chemical structure, concentration, and 


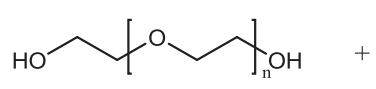

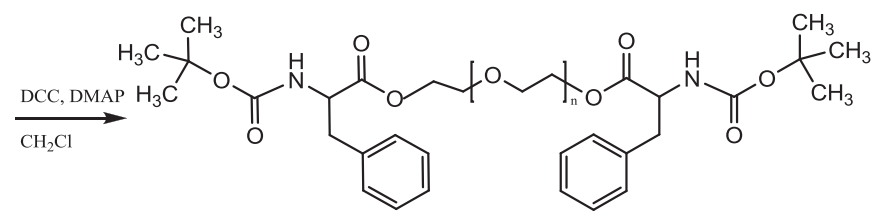

Scheme 1. Synthesis of PEG-8000-phenylalanine conjugate polymer.

molecular weight of the polymer, and temperature have an effect on the behavior of proteins. However, is still difficult to define a priori conditions for the separation of a protein from a given sample. The best conditions for a required purification process are usually found by systematic variation of several factors until a good (not necessarily the best) result is obtained.

Among the ATPS most widely used for the separation of biomolecules are those formed by two polymers, typically polyethylene glycol (PEG)/Dextran or Dextran/Ficoll [7-10]. Although, due to the high cost of some polymer (e.g. Dextran), these systems have been considered to be less economically feasible than polymer/salt ATPS in industrial applications. Also the high viscosity of the polymer phase makes them difficult to handle. In polymer/salt systems, PEG has been the most commonly used polymer due to its biocompatibility, low cost and relatively low viscosity, being predominantly combined with phosphate, sulfate or citrate salts [10-15].

In this work, the effect of changing phase characteristics on protein partition was addressed. For this purpose, PEG was substituted partially or totally by a PEG-amino conjugate in PEG/salt ATPS. Amino acids, being the biological building blocks of proteins, are privileged structures to interact (specifically or non-specifically) with proteins via van der Waals interactions, hydrophobic interactions, hydrogen bonding, and electrostatic interactions, among others. In the present study, PEG with average molecular weight of 8000 was used as a model polymer and chemically modified by adding an amino acid (phenylalanine that bears an aromatic side chain) in its hydroxyl terminal. The physical-chemical characterization of the new systems, formed by the modified polymer and potassium phosphate buffer (KPB), $\mathrm{pH} 7.2$, was carried out and phase diagrams were obtained at $298.15 \mathrm{~K}$. Following this, the partitioning behavior of lysozyme (Lyz) and bovine serum albumin (BSA) in an ATPS composed of $17 \%(w / w)$ total polymer and $12.5 \%(\mathrm{w} / \mathrm{w}) \mathrm{KPB}, \mathrm{pH} 7.2$, was determined. The effect on protein partition caused by the modification introduced in the polymer was evaluated by adding different amounts of PEG-phenylalanine conjugate polymer to the system. The partition coefficient of the PEG-phenylalanine conjugate polymer in these systems was also investigated.

One important factor governing protein partitioning is the hydrophobic character of the equilibrium phases, therefore, the partition coefficients of a series of dinitrophenylated (DNP) amino acids with aliphatic side chain were obtained experimentally and the standard free energy of transfer of a methylene group between the coexisting phases was determined $[9,11,12]$.

The macromolecular structure of proteins and their surface hydrophobicity have a strong influence in the intermolecular interactions [15]. Therefore, fluorescence spectroscopy was used in order to obtain information about the molecular mechanism of interaction with the polymer. The protein surface hydrophobicity (So) was estimated using a fluorescence probe, 8anilinonaphthalene-1-sulfonic acid ammonium salt (ANS) and the relationship between the So and the protein partitioning behavior analyzed. This probe has been widely used for spectrofluorometric measurements namely, to quantify protein hydrophobicity, to monitor conformational changes in biological macromolecules, and to study protein binding sites [16].

\section{Experimental}

\subsection{Chemicals and biologicals}

Lyz from chicken egg white (L6876), BSA (A9647), PEG-8000 with average molecular weight 8000 (Sigma-Aldrich P2139) and ANS, purity 97.0\%, were obtained from Sigma-Aldrich (St. Louis, MO, USA). Dichloromethane (Themo Fisher Scientific, Waltham, MA, USA), N,N'-Dicyclohexylcarbodiimide (DCC) from Sigma-Aldrich, 4-(Dimethylamino)pyridine (DMAP) from Merck and Boc-L-phenylalanine (Boc- $L$-Phe-OH), 99\% purity (Fluka, Sigma-Aldrich), were used. Potassium hydrogen phosphate, $\mathrm{K}_{2} \mathrm{HPO}_{4}, 99.0 \%$ purity was purchased from Merck (Darmstadt, Germany) and potassium dihydrogen phosphate, $\mathrm{KH}_{2} \mathrm{PO}_{4}, 99.0 \%$ purity, supplied by USB Corporation (Cleveland, OH, USA). KPB was prepared using $\mathrm{K}_{2} \mathrm{HPO}_{4}$ and $\mathrm{KH}_{2} \mathrm{PO}_{4}$. To achieve the desired $\mathrm{pH}$ value, different ratio of monobasic and dibasic salts was used. The DNP amino acids were purchased from Sigma-Aldrich: $\mathrm{N}$-(2,4-dinitrophenyl)-glycine (DNP-Gly), N-(2,4-dinitrophenyl)L-alanine (DNP-Ala), N-(2,4-dinitrophenyl)-DL-norvaline (DNPNVal), N-(2,4-dinitrophenyl)-DL-norleucine (DNP-NLeu), N-(2,4dinitrophenyl)-DL- $\alpha$-amino-n-caprylic acid (DNP-AO). All products were used as received without further purification.

\subsection{Synthesis of PEG-8000-phenylalanine conjugate polymer}

The conjugate polymer was synthesized by standard ester formation chemistry using DCC as coupling reagent and DMAP as catalyst (Scheme 1 ). In a typical procedure, to an ice-cooled solution of PEG-8000 (20.0 g, $5.0 \mathrm{mmol} \mathrm{OH}$ terminal groups) in $\mathrm{CH}_{2} \mathrm{Cl}_{2}$ $(40 \mathrm{~mL})$ was sequentially added Boc- $L-\mathrm{Phe}-\mathrm{OH}(1.59 \mathrm{~g}, 6.0 \mathrm{mmol})$, DMAP $(0.060 \mathrm{~g}, 0.5 \mathrm{mmol})$ and a solution of DCC $(1.24 \mathrm{~g}, 6.0 \mathrm{mmol})$ in $\mathrm{CH}_{2} \mathrm{Cl}_{2}(5 \mathrm{~mL})$. The reaction mixture was stirred at the ice bath temperature for $2 \mathrm{~h}$, allowed to reach room temperature and further stirred for $16 \mathrm{~h}$. The Dicyclohexylurea precipitate was removed by filtration and the dichloromethane solution was concentrated at reduced pressure to approximately $20 \mathrm{~mL}$. The reaction product was precipitated by adding diethyl ether $(\sim 200 \mathrm{~mL})$ to the stirring dichloromethane solution. The copious white solid was removed by filtration, dissolved in dichloromethane $(\sim 20 \mathrm{~mL})$ and precipitated again by adding diethyl ether. This procedure was repeated twice and the solid was dried at room temperature (18.4 g).

The derivatized PEG was characterized by ${ }^{1} \mathrm{H}$ and ${ }^{13} \mathrm{C}$ Nuclear Magnetic Resonance Spectroscopy (NMR), Differential Scanning Calorimetry (DSC) and Matrix Assisted Laser Desorption Ionization (MALDI-TOF-MS).

\subsection{Phase diagrams}

Phase diagrams for polymer/KPB ( $\mathrm{pH}$ 7.2) systems were obtained experimentally. Systems with PEG-phenylalanine conjugated polymer were prepared using different percentage of the modified polymer in relation to the total polymer. Binodal curve was determined using the cloud-point method [11]. Suitable amounts of 50\% (w/w) PEG-8000 stock solution, $22.75 \%(w / w)$ KPB stock solution, solid PEG-phenylalanine conjugate polymer, and water were mixed in tubes to obtain a heterogeneous system 
( $2 \mathrm{~g}$ total weight). The tubes were shaken and kept in a thermostatic bath, at constant temperature (298.15 K), to achieve phase equilibrium (10-40 min). Afterwards, known amounts of water (10-100 mg) were added to the tube until a homogeneous system was obtained. All weighing was carried out on an analytical balance precise to within $\pm 0.2 \mathrm{mg}$ (OHAUS, Explorer Pro, Model EP214DC, Switzerland). The binodal curve was determined for each system using different initial masses of polymer and salt stock solutions and adjusted to the following empirical equation [17]:

$y=a \cdot \exp \left(b \cdot x^{0.5}-c \cdot x^{3}\right)$

where $y$ and $x$ are the polymer and salt compositions in mass fraction, respectively, and $a, b$, and $c$ are adjustable parameters without any clear physical meaning.

\subsection{Partitioning studies}

\subsubsection{Biphasic}

ATPS consisting of $17 \%(\mathrm{w} / \mathrm{w})$ total polymer and $12.5 \%(\mathrm{w} / \mathrm{w})$ $\mathrm{KPB}, \mathrm{pH} 7.2$, were prepared in $15 \mathrm{~mL}$ graduated centrifuge tubes by weighting an appropriate amount of PEG-8000 stock solution (50\%, w/w), solid PEG-phenylalanine conjugate polymer and stock solution of KPB $(22.75 \%, w / w)$. To this mixture, adequate water was added to achieve a final mass of $2 \mathrm{~g}$. The mixtures were vortex mixed and allowed to settle for $24 \mathrm{~h}$. Samples were withdrawn from separated phases and after appropriate dilution the total content of modified PEG in both phases was quantified by fluorescence with $\lambda_{\text {ex }} 260 \mathrm{~nm}$ and $\lambda_{\text {em }} 282 \mathrm{~nm}$ (accuracy $= \pm 0.5 \mathrm{~nm}$, Horiba Jobin Yvon FluorMax 4$)$. The partition coefficient $(K)$ is defined as the ratio of the solute concentration in the top (polymer) and bottom (salt) enriched phases. To determined each $K$, three to five partition experiments were carried out at the specified polymer and salt composition of the system, taking into consideration the corresponding dilution factors used in the experiment. In all cases, only the deviation from the average $K$ value below $5 \%$ was accepted.

\subsubsection{Protein partition}

Protein solutions were prepared in water at concentration of $1 \mathrm{mg} \mathrm{mL}^{-1}$. Partitioning studies were performed by adding different amounts of protein solution to six ATPS replicates, in order to get a final concentration ranging from 20 to $100 \mu \mathrm{g}$. Each system has the same ATPS composition (17\%, w/w total polymer/12.5\%, w/w KPB) but different protein concentration, to ensure that protein aggregation effects were avoided. For both proteins, the components were vortex mixed for $2 \mathrm{~min}$ and centrifuged at $2830 \times \mathrm{g}$ for $20 \mathrm{~min}$ to ensure complete phase separation $[11,18]$. After that, three samples of each phase were withdrawn and conveniently diluted for solute quantification. The average of the three measurements was considered (standard deviations lower than $6 \%$ was achieved in all cases). The bichinchoninic acid (BCA) [19] method was used to quantify the concentration of Lyz. The total BSA content in both top and bottom phases was determined using the Bradford method [20]. The partition coefficients determined correspond to the slope of the straight line obtained when comparing the solute concentration in the top phase against that in the bottom phase, for the six replicates prepared, corrected with the corresponding dilution factors $(D F)$ :

$K=\frac{\left[\text { Conc. }(\text { top }) \cdot D F_{\text {top }}\right]}{\left[\text { Conc. }(\text { bottom }) \cdot D F_{\text {bottom }}\right]}$

\subsubsection{DNP amino acids partition}

DNP amino acids solutions of $10 \mathrm{mM}$ were prepared in $10 \mathrm{mM}$ $\mathrm{NaOH}$ and the exposure to light minimized. The partition coefficients were measured experimentally from the tie-line mentioned before. Each DNP amino acid was dosed at varied concentrations and the corresponding amount of water added to each system for a final weight $500 \mathrm{mg}( \pm 0.2 \mathrm{mg}$, OHAUS, Explorer Pro, Model EP214DC, Switzerland). The tubes were thoroughly mixed on a vortex mixer for $2 \mathrm{~min}$, and then the phases were resolved using centrifugation $(2830 \times \mathrm{g}$ for $20 \mathrm{~min})$. Samples of each phase were withdrawn and conveniently diluted, and the absorbance at $362 \mathrm{~nm}$ was measured on a UV-vis spectrometer (ELISA reader, Biotech, Synergy HT, USA). The partition coefficient was calculated as the slope of the straight line obtained when comparing the concentration in the top phase against that in the bottom phase, corrected with the corresponding dilution factor (Eq. (2)) [9,11,12]. Blank systems at corresponding dilutions were used for comparison.

\subsection{Measurement of the protein surface hydrophobicity (So)}

The protein surface hydrophobicity was determined applying a fluorescence probe method using ANS [21]. Stock solutions of $2 \mathrm{mM}$ ANS and $0.1 \mathrm{mM}$ protein were prepared in KPB $(50 \mathrm{mM}, \mathrm{pH} 7.4)$. Fresh solutions were used and the exposure to light minimized by wrapping the containers in aluminum foil. The range of polymer concentration used was $5-20 \%(w / w)$. Aliquots $(1-100 \mu \mathrm{L})$ of the protein solution and $10 \mu \mathrm{L}$ of ANS were added to successive samples containing $1 \mathrm{~mL}$ of buffer solution or polymer solution. The final samples with a constant probe concentration $(20 \mu \mathrm{M})$ and a protein concentration varying from 0 to $100 \mu \mathrm{M}$ were vortex mixed. The fluorescence emission intensities at $470 \mathrm{~nm}$ (while exciting at $360 \mathrm{~nm}$ ) were measured in an ELISA reader (Biotech, Synergy HT, USA). Only when bounded to the protein the ANS acquire fluorescence emission, being the fluorescence of free ANS negligible. Each system was run at least in duplicate and no time dependence of the fluorescence was observed within 30-90 min after mixing. Under the foregoing conditions, with excess probe, the initial slope (So) of the fluorescence intensity versus protein concentration plot has been shown to be correlated to protein surface hydrophobicity [16].

\section{Results}

\subsection{Synthesis of PEG-8000-phenylalanine conjugate polymer}

Successful derivatization of PEG-8000 with phenylalanine was achieved as described in Section 2. Purification of the product was performed by repeated precipitation, from dichloromethane solution, by the addition of diethyl ether. DSC analysis of the starting PEG-8000 and the reaction product reveals an endothermic processes (plausibly melting) at $337.15 \mathrm{~K}$ and $330.15 \mathrm{~K}$, for PEG-8000 and for the reaction product, respectively.

The purity of the product and conversion degree were assessed by proton NMR and MALDI-TOF-MS. Analysis of the ${ }^{1} \mathrm{H}$ and ${ }^{13} \mathrm{C}$ NMR spectra of the reaction product suggests that unreacted starting amino acid (Boc- $L$-Phe-OH), coupling reagent (DCC) and its bi-product (DCU), and catalyst (DMAP) have all been quantitatively removed. The percentage of conversion (bis-derivative) was estimated by ${ }^{1} \mathrm{H}$ NMR and MALDI as $\sim 83 \%$.

\subsection{Phase diagrams}

The experimental binodal curve obtained for the PEG-8000/KPB $(\mathrm{pH} 7.2)$ ATPS is presented in Fig. 1. The phase diagrams for the systems PEG-KPB substituted with different percentages of PEG-phenylalanine conjugate polymer, in relation to the total polymer, are also shown in Fig. 1. The binodal curve is shifted to the origin with the increase in \% of conjugate polymer, increasing the area of the two-phase region. The observed effect is very small for $10-30 \%$ of modified polymer but is more pronounced for $50-100 \%$.

The binodal curves are represented by best-fit lines using the empirical equation suggested by Merchuk and co-workers [17]. The 
Table 1

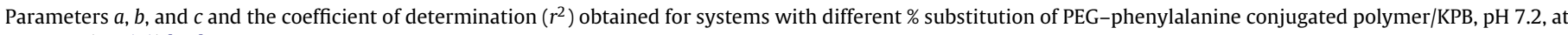
298.15 K (Eq. (1)) [17].

\begin{tabular}{|c|c|c|c|c|}
\hline PEG-phenylalanine conjugate polymer (\%) & $a$ & $b$ & $c$ & $r^{2}$ \\
\hline 0 & $1.005 \pm 0.024$ & $-6.441 \pm 0.138$ & $811.9 \pm 34.6$ & 0.9996 \\
\hline 10 & $1.009 \pm 0.027$ & $-6.386 \pm 0.159$ & $944.2 \pm 46.1$ & 0.9994 \\
\hline 30 & $0.946 \pm 0.028$ & $-6.063 \pm 0.179$ & $1278.4 \pm 58.6$ & 0.9994 \\
\hline 50 & $0.937 \pm 0.035$ & $-6.116 \pm 0.235$ & $1633.3 \pm 89.2$ & 0.9991 \\
\hline 100 & $0.965 \pm 0.035$ & $-6.555 \pm 0.235$ & $2440.1 \pm 108.4$ & 0.9991 \\
\hline
\end{tabular}

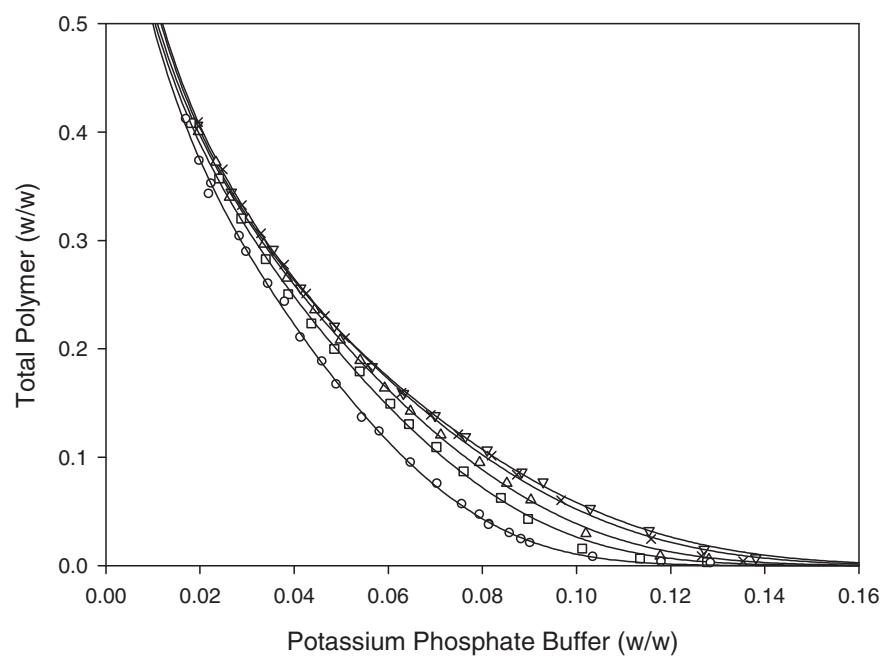

Fig. 1. Experimentally determined ATPS phase diagrams (mass fraction) for different \% substitution of PEG-phenylalanine conjugated polymer in PEG-8000/KPB systems at $298.15 \mathrm{~K}:(\nabla)$ PEG-8000, $(\times) 10 \%,(\triangle) 30 \%$, $(\square) 50 \%$, and $(\bigcirc) 100 \%$ of conjugated PEG, respectively.

parameters of Eq. (1) and the respective coefficients of determination $\left(r^{2}\right)$ are given in Table 1 .

\subsection{Partitioning}

\subsubsection{PEG-8000-phenylalanine conjugate polymer partition}

Fig. 2 presents the PEG-phenylalanine conjugated polymer concentration $\left(\mathrm{mg} \mathrm{mL}^{-1}\right)$, in the top and bottom phase, corrected with the corresponding dilution factor $(D F)$. For concentrations below $20 \%$ it was not possible to quantify the concentration of the solute

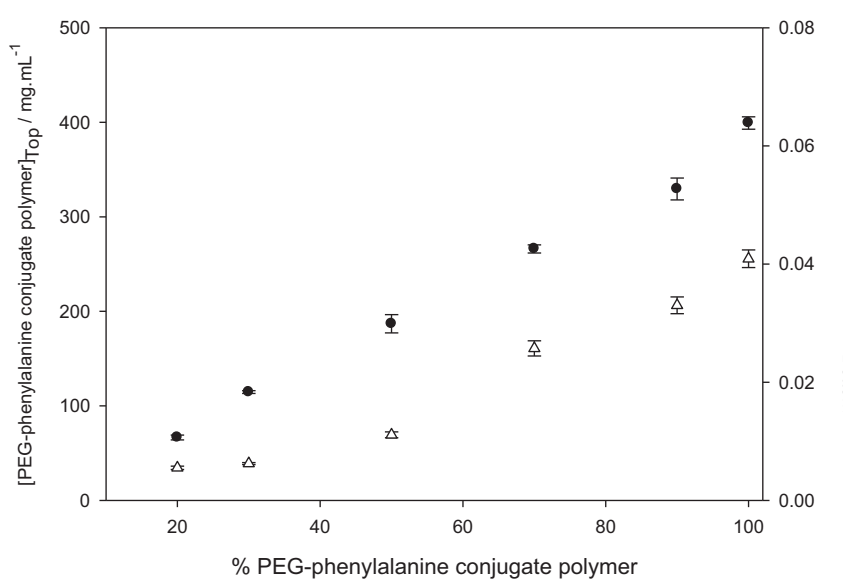

Fig. 2. PEG-phenylalanine conjugated polymer concentration $\left(\mathrm{mg} \mathrm{mL}^{-1}\right)$, in the top phase $(\bullet)$ and bottom phase $(\triangle)$, corrected with the corresponding dilution factor $(D F)$, in PEG-8000/KPB system containing different amounts of PEG-phenylalanine conjugated polymer. ATPS composed of $17 \%(\mathrm{w} / \mathrm{w})$ total polymer and $12.5 \%(\mathrm{w} / \mathrm{w})$ $\mathrm{KPB}, \mathrm{pH} 7.2$.

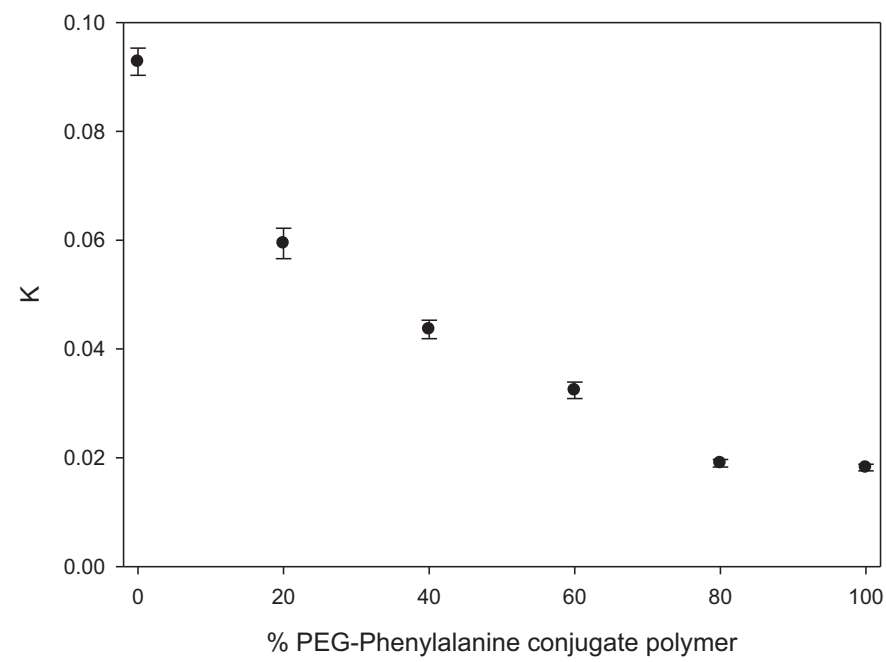

Fig. 3. Partitioning of Lyz in PEG-8000/KPB system containing different substitution of PEG-phenylalanine conjugated polymer. ATPS containing $17 \%(\mathrm{w} / \mathrm{w})$ total polymer and $12.5 \%(\mathrm{w} / \mathrm{w}) \mathrm{KPB}, \mathrm{pH} 7.2$.

in the bottom (salt) enriched phase. The results show that from 20 to $30 \%$ of modified polymer the $K$ increases around 1.5 times decreasing 1.6 times from 50 to $70 \%$ of modified polymer.

\subsubsection{Protein partition}

Figs. 3 and 4 show the partition coefficient for each protein as a function of conjugate polymer percentage substitution. For Lyz a decrease of partition coefficient with the increase of polymer substitution is observed. In the case of BSA it is possible to observe a significant decrease in the partition for up to $30 \%$ of substituted

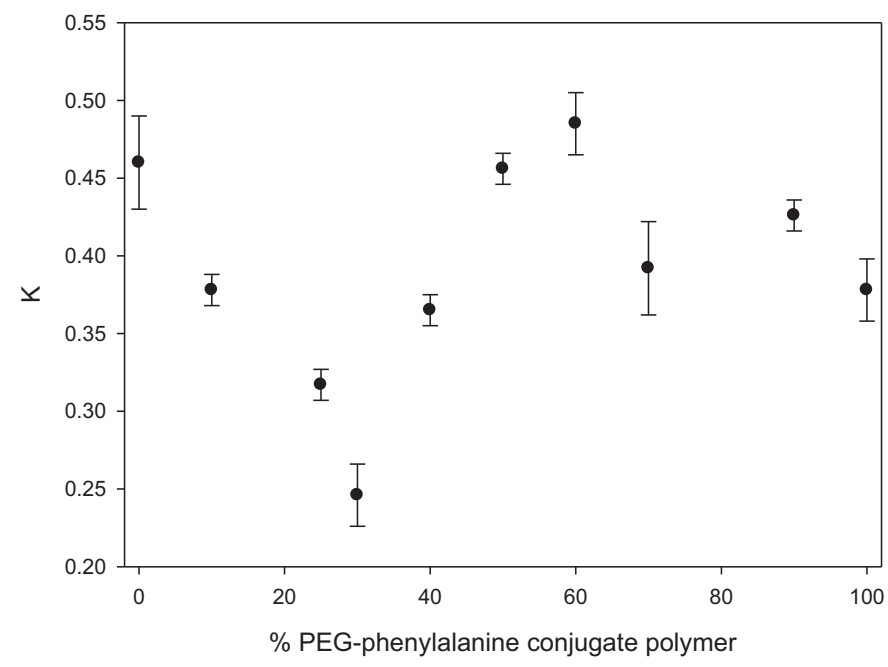

Fig. 4. Partitioning of BSA in PEG-8000/KPB system containing different substitution of PEG-phenylalanine conjugated polymer. ATPS composed of $17 \%(w / w)$ total polymer and $12.5 \%(\mathrm{w} / \mathrm{w}) \mathrm{KPB}, \mathrm{pH} 7.2$. 
Table 2

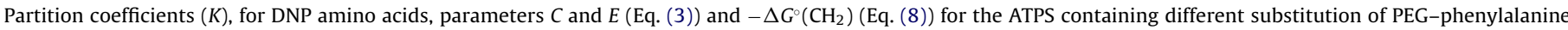
conjugated polymer amounts (\%). ATPS composed of $17 \%(\mathrm{w} / \mathrm{w})$ total polymer and $12.5 \%(\mathrm{w} / \mathrm{w}) \mathrm{KPB}, \mathrm{pH} 7.2$.

\begin{tabular}{|c|c|c|c|c|c|}
\hline & \multicolumn{5}{|c|}{ PEG-phenylalanine conjugate polymer (\%) } \\
\hline & 0 & 10 & 30 & 50 & 100 \\
\hline$-\Delta G^{\circ}\left(\mathrm{CH}_{2}\right)\left(\mathrm{kcal} \mathrm{mol}^{-1}\right)$ & 0.228 & 0.239 & 0.259 & 0.259 & 0.241 \\
\hline$E$ & $0.388 \pm 0.005$ & $0.406 \pm 0.020$ & $0.441 \pm 0.010$ & $0.440 \pm 0.007$ & $0.410 \pm 0.031$ \\
\hline$C$ & $2.25 \pm 0.02$ & $2.35 \pm 0.07$ & $2.36 \pm 0.04$ & $2.39 \pm 0.02$ & $2.98 \pm 0.11$ \\
\hline$r^{2}$ & 0.999 & 0.995 & 0.998 & 1.000 & 0.984 \\
\hline K (DNP-Gly) & $11.63 \pm 0.04$ & $12.79 \pm 0.13$ & $13.93 \pm 0.23$ & $14.56 \pm 0.25$ & $26.57 \pm 0.53$ \\
\hline$K($ DNP-Ala $)$ & $15.93 \pm 0.16$ & $17.71 \pm 0.59$ & $18.12 \pm 0.58$ & $19.08 \pm 0.58$ & $37.06 \pm 0.69$ \\
\hline$K($ DNP-NVal $)$ & $27.10 \pm 0.65$ & $38.52 \pm 0.69$ & $34.59 \pm 1.38$ & $57.25 \pm 0.58$ & $51.17 \pm 1.00$ \\
\hline$K($ DNP-NLeu $)$ & $39.80 \pm 1.16$ & $53.41 \pm 1.20$ & $58.86 \pm 2.83$ & $87.90 \pm 0.97$ & $80.50 \pm 2.01$ \\
\hline$K(\mathrm{DNP}-\mathrm{AO})$ & $108.07 \pm 3.42$ & $127.71 \pm 4.94$ & $165.55 \pm 6.43$ & $176.08 \pm 8.21$ & $288.36 \pm 15.93$ \\
\hline
\end{tabular}

polymer, followed by an inverse trend in favor of the rich polymer phase up to $60 \%$. For higher values a moderate variation of the distribution coefficients is observed.

\subsubsection{DNP amino acids partition}

The experimental partition coefficients of DNP amino acids using an ATPS composed of $17 \%(\mathrm{w} / \mathrm{w})$ total polymer and $12.5 \%$ $(\mathrm{w} / \mathrm{w}) \mathrm{KPB}, \mathrm{pH} 7.2$, are given in Table 2 . The relationship experimentally obtained for the logarithm of the $K$ and the number of equivalent methylene groups in the aliphatic side chain of homologous DNP amino acids partitioned in the ATPS examined can be described as:

$\ln K=C+E \cdot n\left(\mathrm{CH}_{2}\right)$

where $n\left(\mathrm{CH}_{2}\right)$ is the average equivalent number of methylene $\left(\mathrm{CH}_{2}\right)$ groups in the amino acid aliphatic side chain and $C$ and $E$ are constants. The physical meaning of both parameters $C$ and $E$ was discussed in detail elsewhere $[9,11,12]$. These two parameters, calculated by linear regression, and the corresponding coefficients of determination $\left(r^{2}\right)$ are presented in Table 2. Typical example of experimental results are shown as logarithms of the distribution coefficients plotted against the $n\left(\mathrm{CH}_{2}\right)$ in a given ATPS (Fig. 5).

\subsubsection{Measurement of the protein surface hydrophobicity (So)}

The effect of the concentration of PEG-8000 and PEG-phenylalanine conjugate polymer on the So of BSA is shown in Fig. 6. So value was calculated as the initial slope of the

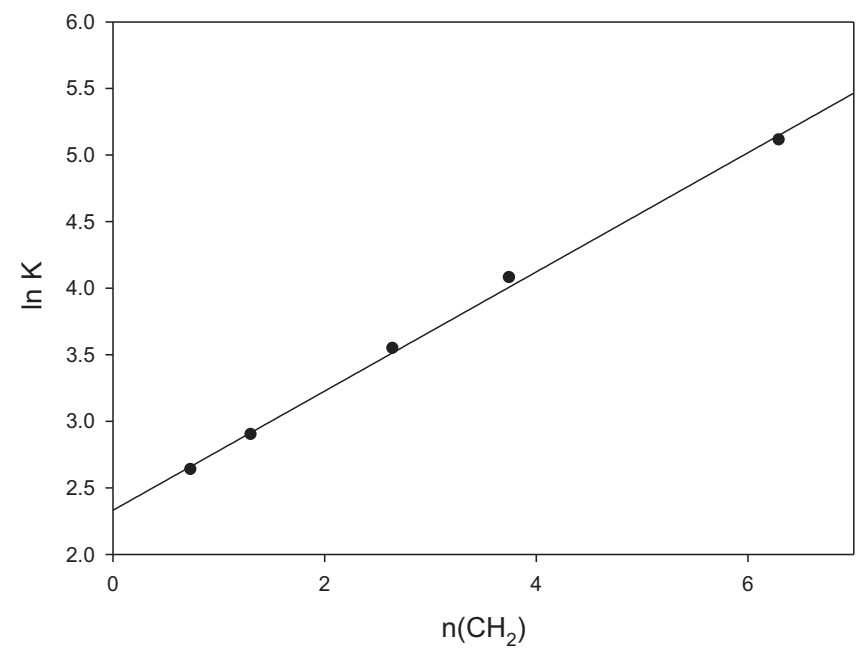

Fig. 5. Logarithm of the distribution coefficients, $K$, for homologous series of DNP amino acids partitioned as a function of the average equivalent number of methylene $\mathrm{CH}_{2}$ groups, $n\left(\mathrm{CH}_{2}\right)$, using an ATPS composed of $17 \%(\mathrm{w} / \mathrm{w})$ total polymer, being $30 \%$ of PEG-phenylalanine conjugated polymer, and $12.5 \%$ (w/w) KPB, pH 7.2. titration curves of the fluorescence probe ANS as function of the protein concentration (curves not shown). A considerable decrease in So is observed with the increase of the concentration of both polymers. In addition the So is considerably lower in the presence of the modified PEG.

\section{Discussion}

Liquid-liquid equilibrium data for the polymer/KPB ( $\mathrm{pH} 7.2$ ) ATPSs (Fig. 1) was successfully adjusted to the empirical equation suggested by Merchuk and co-workers [17]. The coefficients of determination $\left(r^{2}\right)$ obtained, are in all cases greater than 0.999 (see Table 1). It was found that the coefficients $a, b$, and $c$ (Eq. (1)) could be related with the percentage of substituted PEG-conjugate $(N)$ as follows:

$\frac{c}{a}=776.5105+20.2538 N-0.0269 N^{2} \quad\left(r^{2}=0.9984\right)$

$\frac{c}{b}=-121.9744-3.1937 N+0.0069 N^{2} \quad\left(r^{2}=0.9987\right)$

$c=796.0984+16.4604 N \quad\left(r^{2}=0.9995\right)$

As expected, the chemical structure and composition of the polymer has a significant effect on the phase separation. For $10 \%$ substituted PEG the effect appears to be small but with the increase of PEG conjugate (\%) present in the ATPS, the binodal curve was shifted towards the origin and the biphasic formation occurs at lower concentrations of polymer and salt. The more hydrophobic character of the modified PEG, due to the introduction of the

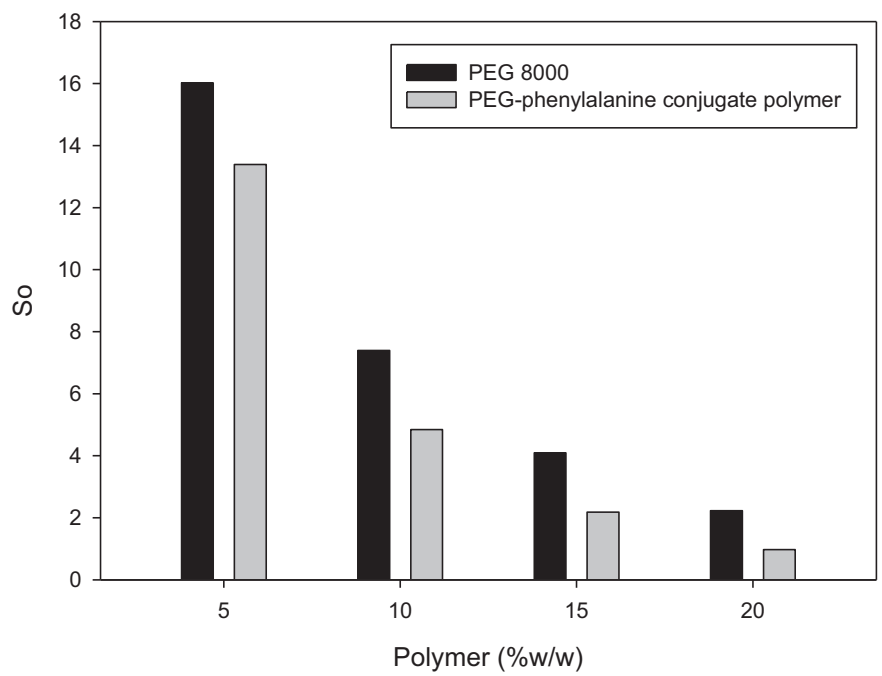

Fig. 6. Effect of polymer concentration on the BSA surface hydrophobicity. 
amino acid phenylalanine, increases the incompatibility between the system components.

However, the distribution of the modified polymer between the two phases does not remain constant with the increase in its concentration, as would be expected for a true partitioning behaviour. Analysis of PEG-phenylalanine conjugated concentration in both phases (Fig. 2) reveal that whereas in the top phase it increases linearly with percentage substitution, in the bottom phase this increase is approximately exponential until $70 \%$ substituted PEG, following a linear variation for higher values. Given that PEG-phenylalanine conjugate concentration in the bottom phase is about four orders of magnitude lower than the concentration in the upper phase this suggests the saturation of the top phase with the modified polymer for higher concentrations.

Lyz partitioning follows the expected trend, decreasing with the increase of PEG-phenylalanine concentration in the system. In fact, according to Fig. 1, for systems with the same total composition of polymer and salt, the increase of PEG-conjugate correspond to an increase of the tie-line length, e.g. an increase of total phase composition difference. Previous results show that partitioning in ATPS is linearly dependent on the difference between the concentration of polymer in the two phases [10].

However, BSA does not follow the same trend and its partition profile is approximately symmetrical to the one observed for the partition of the modified PEG. This result is unexpected but the extreme sensitivity to phase composition of BSA must be related with its higher molecular weight $(67,000 \mathrm{Da})$ compared with Lyz $(14,400 \mathrm{Da})$. It is well known that partition in liquid-liquid systems depends exponentially with solute mass by [22]:

$\ln K=\frac{\lambda M}{k T}$

where $K$ is partition coefficient, $M$ is the molecular mass of the solute, $k$ is the Boltzmann constant, $T$ is the absolute temperature, $\lambda$ is the parameter that accounts for all the factors that determine partition.

In this case, a small alteration of phase hydrophobicity may be related to the observed behavior. Determination of the relative hydrophobicity of the phases (E, Eq. (3)) shed some light on this issue. This parameter is related with the standard free energy of transfer of the methylene group from one phase to the other in a given ATPS, $-\Delta G^{\circ}\left(\mathrm{CH}_{2}\right)$ :

$\Delta G^{\circ}\left(\mathrm{CH}_{2}\right)=-R T E$

where $R$ is the universal gas constant, $T$ is the absolute temperature in Kelvin (see Table 2). The value of $-\Delta G^{\circ}\left(\mathrm{CH}_{2}\right)$ for the system without modified PEG is less negative than the corresponding values for systems containing conjugate polymer. Such trend confirms that the conjugate polymer-rich phases are, as expected due to chemical modification, more hydrophobic. This fact confirms the results presented in Section 3.2. It should be noted that the difference observed is more pronounced in the system with $30 \%$ of modified PEG and decreasing after. However, the difference does not allow us to establish a clear relationship between the relative hydrophobicty of the phases and the BSA partitioning behavior.

In order to obtain more information about the molecular mechanism of the multiple interactions polymer-water-protein, a fluorescence method, using ANS as probe, was used to determine So [21]. By applying this method, an important modification in the BSA surface hydrophobicity was observed for both polymers, this effect being more noticeable for the polymer of higher hydrophobic character, which in fact is the modified PEG (Fig. 6). The So values for Lyz could not be assayed using this methodology because interaction of Lyz with ANS is very weak (data not shown). This suggests that Lyz does not have adequate hydrophobic zones to bind this probe. However, according to previous literature results [23],
Lyz hydrophobicity, calculated by the inverse of the ammonium sulphate concentration needed to precipitate a $2 \mathrm{~g} \mathrm{~L}^{-1}$ solution of the protein, is higher than BSA. Taking into account that the same concentration (expressed as mass per volume) corresponds to a higher concentration in Molar, the protein with the lower molecular weight (Lyz) is 4.65-fold more concentrated then BSA what might explain the results obtain.

From our data it is clear that BSA is more hydrophobic then Lys and this taken together with is higher molecular weight explain the different behavior of the two proteins with the increase of PEG-phenylalanine in the systems.

Proteins with great hydrophobic surface area exposed to solvent and have the possibility of interacting with PEG. Although it is shown that both polymers showed to perturb the structure of BSA surface, no clear relationship could be established between the partitioning behavior and So.

\section{Conclusions}

The influence of chemical structure in the formation of ATPS and partition of proteins was assessed in this study. PEG-8000 was used as a model polymer and modified by introducing phenylalanine in its hydroxyl terminals. Liquid-liquid equilibrium data for different substitution of PEG-amino acid conjugate polymer in PEG-8000/KPB (pH 7.2) ATPSs was determined at 298.15 K. An important shift in the position of the binodal line was observed with the increase of PEG-phenylalanine conjugated polymer.

However, the partition of PEG-phenylalanine conjugated does not remain constant with the increase of modified polymer substitution reaching a maximum for $30 \%$ and then decreasing until complete substitution.

Different behavior was observed for the two proteins studied, BSA and Lyz. Whereas partition of Lyz decreases with the increase of modified polymer substitution, the partition of BSA is approximately symmetrical to the one observed for the PEG-phenylalanine conjugate. This indicates an extreme sensibility of BSA to a small change of phase hydrophobicity observed for intermediate values of polymer substitution. The higher molecular weight of BSA compared to Lyz and also its higher hydrophobicity should be the factors that justify these results. Additional studies for phase and proteins surface hydrophobicity further corroborate this hypothesis.

Our findings highlight the importance of proteins structure in ATPS partition and also the different sensibility of proteins to changes in the chemical structure of the ATPS forming polymers.

\section{Acknowledgments}

L.A. Ferreira and S.C. Silvério acknowledge the financial support (Grants SFRH/BPD/47607/2008 and SFRH/BD/43439/2008, respectively) from Fundação para a Ciência e a Tecnologia (Lisboa, Portugal).

\section{References}

[1] K. Selber, F. Tjerneld, A. Collén, T. Hyytiä, T. Nakari-Setälä, M. Bailey, R. Fagerström, J. Kan, J. van der Laan, M. Penttilä, M. Kula, Process Biochem. 39 (2004) 889-896.

[2] P.A.J. Rosa, A.M. Azevedo, S. Sommerfeld, M. Mutter, M.R. Aires-Barros, W. Backer, J. Biotechnol. 139 (2009) 306-313.

[3] K. Naganagouda, V.H. Mulimani, Process Biochem. 43 (2008) 1293-1299.

[4] J.T. Hsu, US Patent 4,980,065 (1990).

[5] P.A.J. Rosa, A.M. Azevedo, S. Sommerfeld, W. Backer, M.R. Aires-Barros, Biotechnol. Adv. 29 (2011) 559-567.

[6] O. Aguilar, V. Albiter, L. Serrano-Carreón, M. Rito-Palomares, J. Chromatogr. B 835 (2006) 77-83.

[7] P.P. Madeira, J.A. Teixeira, E.A. Macedo, L.M. Mikheeva, B.Y. Zaslavsky, Fluid Phase Equilib. 267 (2008) 150-157.

[8] P.P. Madeira, J.A. Teixeira, E.A. Macedo, L.M. Mikheeva, B.Y. Zaslavsky, J. Chromatogr. A 1190 (2008) 39-43. 
[9] P.P. Madeira, J.A. Teixeira, E.A. Macedo, J. Chromatogr. A 1185 (2008) 85-92.

[10] B.Y. Zaslavsky, Aqueous Two-phase Partitioning: Physical Chemistry and Bioanalytical Applications, Marcel Dekker, New York, 1994.

[11] S.C. Silvério, P.P. Madeira, O. Rodríguez, J.A. Teixeira, E.A. Macedo, J. Chem. Eng. Data 53 (2008) 1622-1625.

[12] O. Rodríguez, S.C. Silvério, P.P. Madeira, J.A. Teixeira, E.A. Macedo, Ind. Eng. Chem. Res. 46 (2007) 8199-8204

[13] J.G. Huddleston, H.D. Willauer, R.D. Rogers, J. Chem. Eng. Data 48 (2003) $1230-1236$

[14] A.M. Azevedo, A.G. Gomes, P.A.J. Rosa, I.F. Ferreira, A.M.M.O. Pisco, M.R. AiresBarros, Sep. Purif. Technol. 65 (2009) 14-21.

[15] A.M. Azevedo, P.A.J. Rosa, I.F. Ferreira, M.R. Aires-Barros, J. Biotechnol. 132 (2007) 209-217.
[16] C.A. Haskard, E.C.Y. Li-Chan, J. Agric. Food Chem. 46 (1998) 2671-2677.

[17] J.C. Merchuk, B.A. Andrews, J.A. Asenjo, J. Chromatogr. B 711 (1998) 285-293.

[18] S.C. Silvério, O. Rodríguez, J.A. Teixeira, E.A. Macedo, Fluid Phase Equilib. 296 (2010) 173-177.

[19] P.K. Smith, R.I. Krohn, G.T. Hermanson, A.K. Mallia, F.H. Gartner, M.D. Provenzano, E.K. Fujimoto, N.M. Goeke, B.J. Olson, D.C. Klenk, Anal. Biochem. 150 (1985) 76-85.

[20] M.M. Bradford, Anal. Biochem. 72 (1976) 248-254.

[21] G. Tubío, B. Nerli, G. Picó, J. Chromatogr. B 799 (2004) 293-301.

[22] P.A. Albertsson, Partition of Cell Particles and Macromolecules, 3rd ed., Wiley, New York, 1986.

[23] B.A. Andrews, A.S. Schmidt, J.A. Asenjo, Biotechnol. Bioeng. 90 (2005) 380-390. 